\title{
Kidney dendritic cell activation is required for progression of renal disease in a mouse model of glomerular injury
}

\author{
Felix Heymann, ${ }^{1}$ Catherine Meyer-Schwesinger, ${ }^{2}$ Emma E. Hamilton-Williams, ${ }^{1,3}$ \\ Linda Hammerich, ${ }^{1}$ Ulf Panzer, ${ }^{2}$ Sylvia Kaden, ${ }^{4}$ Susan E. Quaggin, ${ }^{5}$ \\ Jürgen Floege, ${ }^{5}$ Hermann-Josef Gröne, ${ }^{4}$ and Christian Kurts ${ }^{1}$
}

${ }^{1}$ Institutes for Molecular Medicine and Experimental Immunology, Friedrich-Wilhelms-Universität, Bonn, Germany. ${ }^{2}$ III Medizinische Klinik, Universitätsklinikum Hamburg-Eppendorf, Hamburg, Germany. ${ }^{3}$ Medical Clinic II, RWTH University of Aachen, Aachen, Germany. ${ }^{4}$ Department of Cellular and Molecular Pathology, German Cancer Research Center, Heidelberg, Germany. ${ }^{5}$ Samuel Lunenfeld Research Institute, Toronto, Ontario, Canada.

\begin{abstract}
The progression of kidney disease to renal failure correlates with infiltration of mononuclear immune cells into the tubulointerstitium. These infiltrates contain macrophages, DCs, and T cells, but the role of each cell type in disease progression is unclear. To investigate the underlying immune mechanisms, we generated transgenic mice that selectively expressed the model antigens ovalbumin and hen egg lysozyme in glomerular podocytes (NOH mice). Coinjection of ovalbumin-specific transgenic CD8 ${ }^{+} \mathrm{CTL}$ s and $\mathrm{CD}^{+}{ }^{+} \mathrm{Th}$ cells into $\mathrm{NOH}$ mice resulted in periglomerular mononuclear infiltrates and inflammation of parietal epithelial cells, similar to lesions frequently observed in human chronic glomerulonephritis. Repetitive $T$ cell injections aggravated infiltration and caused progression to structural and functional kidney damage after 4 weeks. Mechanistic analysis revealed that DCs in renal lymph nodes constitutively cross-presented ovalbumin and activated CTLs. These CTLs released further ovalbumin for CTL activation in the lymph nodes and for simultaneous presentation to Th cells by distinct DC subsets residing in the kidney tubulointerstitium. Crosstalk between tubulointerstitial DCs and Th cells resulted in intrarenal cytokine and chemokine production and in recruitment of more CTLs, monocyte-derived DCs, and macrophages. The importance of DCs was established by the fact that DC depletion rapidly resolved established kidney immunopathology. These findings demonstrate that glomerular antigen-specific CTLs and Th cells can jointly induce renal immunopathology and identify kidney DCs as a mechanistic link between glomerular injury and the progression of kidney disease.
\end{abstract}

\section{Introduction}

In most kidney diseases, in particular the different forms of glomerulonephritis (GN), renal function and its prognosis are closely correlated with the extent of tubulointerstitial damage and especially with the extent of mononuclear infiltration of immune cells into this compartment (1). Thus, progression of kidney disease to chronic renal failure critically depends on events in the tubulointerstitium, even in primary GN. Several mechanisms have been proposed to explain how glomerular injury may spread to the tubulointerstitium, for example, podocyte damage leading to tubulointerstitial malperfusion, glomerular filtration leakage into the interstitium, or tubulotoxic effects of proteinuria (2-4). Yet the functional role of the prominent tubulointerstitial mononuclear infiltrate in such spreading and in kidney disease progression is unclear. Nevertheless, immunosuppression is the standard therapy in many types of progressive kidney disease, suggesting that the infiltrating immune cells are important. Remarkably, these infiltrates are often located around inflamed glomeruli in various forms of GN, such as diffuse proliferative and crescentic lupus nephritis (5-7), IgA nephropathy (8), or anti-neutrophil cytoplas-

Conflict of interest: The authors have declared that no conflict of interest exists. Nonstandard abbreviations used: DT, diphtheria toxin; DTR, DT receptor; GN, glomerulonephritis; HEL, hen egg lysozyme; $\mathrm{NOH}$ mice, transgenic mice expressing OVA and HEL under the control of the nephrin promoter in glomerular podocytes; OT-I cell, OVA-specific transgenic CD8 ${ }^{+} \mathrm{CTL}$; OT-II cell, OVA-specific $\mathrm{CD}^{+}{ }^{+}$Th cell; RIP, rat insulin promoter

Citation for this article: J. Clin. Invest. 119:1286-1297 (2009). doi:10.1172/JCI38399. mic antibody-associated GN (9). Periglomerular infiltrates contain macrophages, DCs, and T cells $(5,10,11)$. Macrophages are generally recognized as mediators of kidney damage $(2,12)$. DCs have only recently been identified as an abundant component of the healthy tubulointerstitium (10,13-15). They perform sentinel functions and alert the immune system to acute renal injury via cytokine production and stimulation of proinflammatory $\mathrm{T}$ cells $(16,17)$. We recently showed that kidney DCs attenuate the early phase of crescentic GN (18). Their role in chronic GN and in progression of renal disease is unknown.

The role of $\mathrm{T}$ cells in chronic $\mathrm{GN}$ has been controversial for decades (19-22). Experimental studies proposed a role particularly in crescentic and pauci-immune GN $(2,5,23-28)$. However, the exact mechanisms by which the distinct $T$ cell types contribute to GN are only partially understood. In nephrotoxic nephritis, a model of human crescentic GN, Th type 1 cells specific for a planted glomerular antigen stimulated renal macrophages (27) that caused kidney damage (12). Also CD8 ${ }^{+} \mathrm{T}$ cells (CTLs), whose main function is the defense against intracellular pathogens and tumors, are found in periglomerular infiltrates, e.g., in lupus nephritis (7). Several animal models exist for studying CTLs specific to tubular antigen, and these have facilitated elucidating immune mechanisms in interstitial nephritis (29-31). A role of CTL in GN is supported by the attenuation of rat experimental autoimmune (32) and nephrotoxic nephritis (32) in CD8-knockout mice, albeit murine nephrotoxic nephritis was not ameliorated (27). Thus, the exact role of CTLs in glomerular disease is unclear, mostly because suitable animal models are lacking. 
A

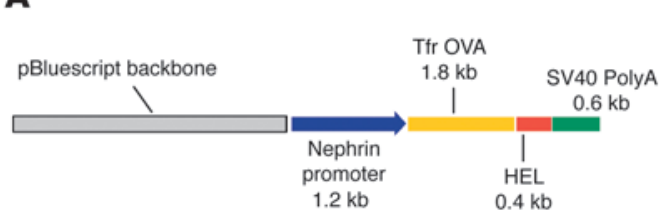

B

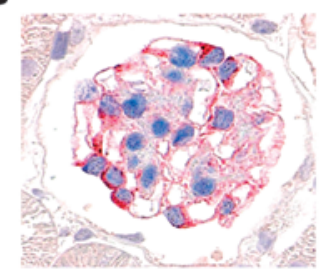

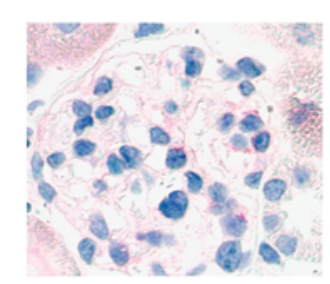

E

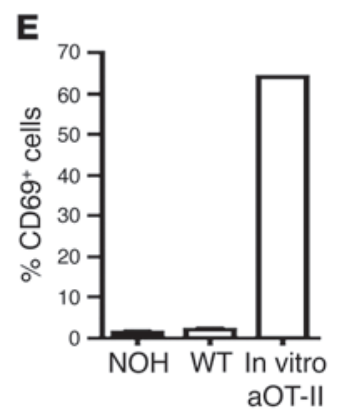

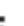

c

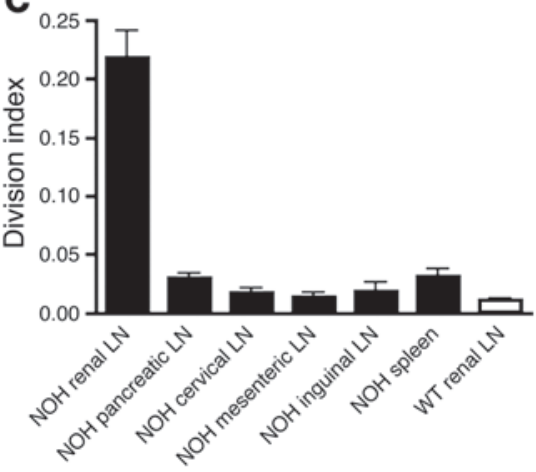

D

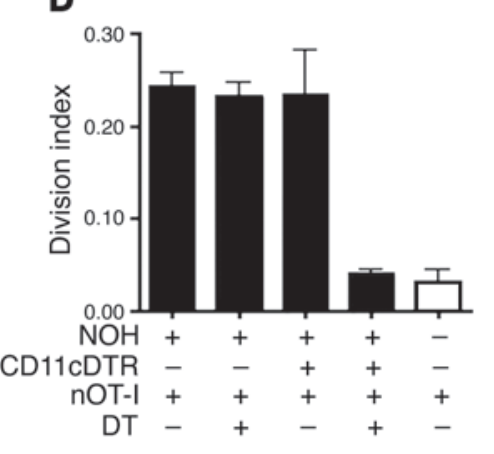

Figure 1

Generation and characterization of mice expressing model autoantigens in glomeruli. (A) Plasmid used to generate $\mathrm{NOH}$ mice. (B) Kidney sections of a NOH (left) and non-Tg control (right) mouse stained for OVA expression. Red, OVA; blue, nuclei. Original magnification, $\times 1,000$. (C) Division indices of CFSE-labeled OT-I cells in various LNs and the spleen of NOH and non-Tg mice on day 3 after adoptive transfer. (D) CFSE-labeled OT-I cells were injected into $\mathrm{NOH} \times \mathrm{CD} 11 \mathrm{c}-\mathrm{DTR}, \mathrm{NOH}$, or non-Tg mice, and DCs were depleted by injection of DT on the same day. Bars indicate division indices of OT-I cells in the renal LN on day 3. nOT-I, naive OT-I. (E) CD69 expression of CFSE-labeled OT-II cells in the renal LN of NOH and non-Tg mice on day 3 after transfer. In vitro-activated OT-II cells were used as positive staining control. Representative flow cytometry data for $\mathbf{C}$ and $\mathbf{D}$ appear in Supplemental Figure 2. Results are representative of 3 experiments in groups of 3 mice. Data are presented as mean \pm SD. aOT-II, activated OT-II cells.

Transgenic models have been key in elucidating the roles of autoreactive CTL and Th cells in immune-mediated disease. In rat insulin promoter-mOVA (RIP-mOVA) mice expressing the model antigen OVA in pancreatic islets and proximal tubules, DCs in draining LNs activated OVA-specific transgenic CD8 ${ }^{+}$CTLs (OT-I cells) by a mechanism termed cross-presentation (33), which is restricted to DCs of the CD8- conventional type (34-39). However, such activation led to peripheral CTL tolerance by deletion, a mechanism also known as cross-tolerance $(40,41)$. Enhancement of cross-presentation by autoantibodies (42) or provision of help by injecting OVA-specific Th cells (OT-II cells) (40) prevented cross-tolerance. Mechanistic analysis revealed that Th cells acted by stimulating cross-presenting DCs in secondary lymphatics to permit cytotoxic effector function, whereas CTLs activated by unlicensed DCs ("help-less" CTLs) were programmed to die after secondary antigen encounters $(43,44)$. Analogous mechanisms were reported in models used to examine different antigens or expression sites (45-47). Furthermore, Th cells may regulate the CTL effector phase in nonlymphatic organs $(34,35,46)$. However, the rapid destruction of pancreatic islets by CTLs precluded elucidation of the underlying mechanisms in models in which antigen was expressed in this site.

To address these open questions, we generated transgenic $\mathrm{NOH}$ mice, in which the model autoantigens OVA and hen egg lysozyme (HEL) were expressed in glomerular podocytes. Injection of OT-I or OT-II cells into these animals allowed study of the immunopathology resulting from glomerular antigen-specific CTLs or Th cells, respectively, in a straightforward manner.
Unexpectedly, our studies identified kidney DCs as a mechanistic link between $\mathrm{T}$ cell-mediated glomerular injury and progression of kidney disease.

\section{Results}

Generation and characterization of mice expressing model autoantigens in glomeruli. To straightforwardly study the role of glomerular antigen-specific $T$ cells in nephritis, we generated mice expressing the model autoantigens OVA and HEL in podocytes. These mice allowed use of the well-characterized transgenic OT-I and OT-II mice, which produce OVA-specific CTLs (OT-I cells) and Th cells (OT-II cells), respectively, as donors for autoreactive $\mathrm{T}$ cells specific for a glomerular antigen. A construct containing the podocyte-specific human nephrin promoter (48) fused to cDNA encoding the transmembrane domain of the transferrin receptor, OVA, and HEL (49) (Figure 1A) was used to generate nephrin-OVA-HEL mice (NOH mice) on the C57BL/ 6 background. Immunohistochemistry revealed OVA expression in glomerular podocytes of $\mathrm{NOH}$ mice (Figure 1B) without expression in other organs, in particular pancreatic islets or the brain, where expression of a reporter driven by the murine nephrin promoter had been reported (50), or in non-Tg controls (Figure 1B and Supplemental Figure 1; supplemental material available online with this article; doi:10.1172/JCI38399DS1). Quantitative RT-PCR detected OVA message in the kidney after 40 cycles, while other tissues remained negative for 50 cycles, indicating that renal expression was at least 1,000 -fold (10 cycles) higher than in other tissues (Supplemental Table 1). 

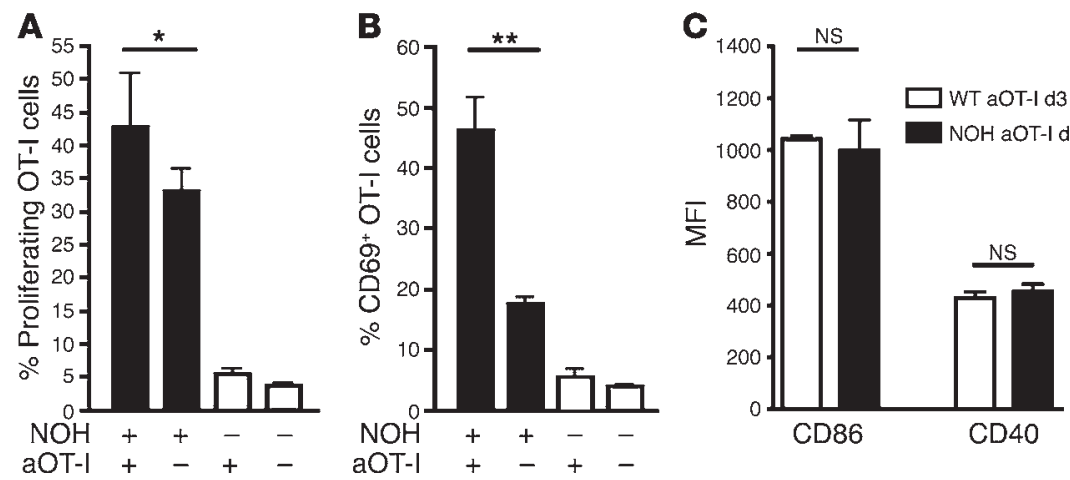

\begin{abstract}
Figure 2
Glomerular antigen-specific CTLs release antigen for cross-presentation in the renal LN. $5 \times 10^{6}$ activated OT-I cells were injected into NOH or WT mice. After 2 days, $2 \times 10^{6} \mathrm{CFSE}$-labeled OT-I cells were injected, and their proliferative response $(\mathbf{A})$ and CD69 expression (B) were determined in the renal LNs. (C) Maturation state of renal LN DCs of $\mathrm{NOH}$ (black bars) and WT mice (white bars) injected with activated OT-I cells was determined by measuring CD86 and CD40 on CD11 $\mathrm{c}^{+}$cells. Representative flow cytometry data appear in Supplemental Figure 4. Results are representative of 3 experiments in groups of 3 mice. ${ }^{*} P<0.05$; ${ }^{* *} P<0.01$. MFI, mean fluorescence intensity.
\end{abstract}

CFSE-labeled OT-I cells have previously been used as sensitive probes for in vivo OVA presentation (33). When these cells were injected i.v. into $\mathrm{NOH}$ mice, they divided only in the renal LN but not in any other LNs nor in the spleen nor in non-Tg control mice (Figure 1C; Supplemental Figure 2A), indicating kidney restriction of $\mathrm{T}$ cell activation and thus of antigen expression. Furthermore, we noted neither neurological symptoms nor diabetes mellitus in $\mathrm{NOH}$ mice injected with OT-I cells, which was the case in mice expressing OVA in pancreatic islets or in neurons, respectively (40, 47). These findings demonstrated that transgene expression in $\mathrm{NOH}$ mice was restricted to kidney podocytes.

To determine whether OT-I activation in the renal LN was a function of DCs, we crossed NOH mice to CD11c-DTR/eGFP (in which DTR indicates diphtheria toxin receptor) mice (here simply termed CD11c-DTR mice), which allow DC deletion by injection of diphtheria toxin (DT) (51). A single DT injection into $\mathrm{NOH} \times \mathrm{CD} 11 \mathrm{c}-$ DTR mice reduced CD $11 c^{+}$cells in the LNs by more than $90 \%$ (Supplemental Figure 2B) and abrogated proliferation of injected OT-I cells in the renal LN (Figure 1D; Supplemental Figure 2B), demonstrating that CD $11 \mathrm{c}^{+}$DCs activated OT-I cells by cross-presentation of glomerular autoantigen. This was not due to differences in the activation state of DCs from $\mathrm{NOH}$ mice, as this was identical to the activation state in non-Tg controls (Supplemental Figure 3).

CFSE-labeled OVA-specific Th cells (OT-II cells) neither proliferated nor expressed the activation marker CD69 after transfer into $\mathrm{NOH}$ mice (Figure 1E). This was consistent with previous studies showing failure of OT-II cells to proliferate in response to OVA expressed as transgenic self antigen and has been explained by their limited affinity in particular for autoantigen $(35,40,46)$. In summary, glomerular autoantigen was constitutively presented by DCs in the renal LNs, but only OT-I cells proliferated in response.

Glomerular antigen-specific CTL release antigen for cross-presentation in the renal LN. To investigate whether activated CTLs caused glomerular damage in our experimental setting, we injected in vitro-activated OT-I cells into NOH mice to induce CTL-mediated glomerular damage. Subsequently, we injected CFSE-labeled naive OT-I cells as in vivo probes for cross-presentation. Indeed, proliferation of CFSE-labeled OT-I cells (Figure 2A; Supplemental Figure 4) and their expression of the activation marker CD69 (Figure 2B; Supplemental Figure 4) in the renal LNs were increased after injection of activated OT-I cells. This was not due to OT-I cell-induced increased expression of costimulatory molecules by renal LN DCs (Figure 2C). Thus, activated CTLs caused the release of podocyte antigen, which was subsequently crosspresented in the renal LNs.
Glomerular antigen-specific CTLs and Th cells jointly induce periglomerular mononuclear infiltration. To determine what type of immunopathology results from glomerular antigen-specific $T$ cells, we injected OT-I and OT-II cells into NOH mice. Neither cell type alone nor a combination of naive OT-I and naive OT-II cells caused any immunopathology. We speculated that this may be due to the lack of constitutive OT-II cell activation in NOH mice (Figure $1 \mathrm{E}$ ). To circumvent this problem, we activated OT-II cells in vitro prior to injection, which has been shown to enable potent in vivo helper functions (35). Activated OT-II cells produced IL-2, TNF- $\alpha$, and IFN- $\gamma$ (data not shown), indicating Th1 differentiation, consistent with previous work using these cells (52). While transfer of activated OT-II cells alone failed to cause immunopathology, their coinjection with naive OT-I cells caused a significant focal periglomerular mononuclear infiltrate in all $\mathrm{NOH}$ recipient mice after 7 days (Figure 3, A and B), as determined by semiquantitative (Figure 3, J and $\mathrm{K}$ ) and quantitative analysis (Figure $3 \mathrm{~L}$ ). Non-Tg recipients did not develop infiltration, indicating antigen specificity (data not shown). Interestingly, intraglomerular infiltration was not detected (Figure 3B). Electron microscopy showed that podocytes maintained regular foot processes, but in contrast to normal podocyte physiology, these cells were in direct contact with parietal cells (Figure 3C). Parietal cells featured large cytoplasm with increased numbers of organelles and were separated by a very thin membrane from the periglomerular infiltrate. Bowman capsule was surrounded by small lymphocytes and monocyte/macrophage-like cells (Figure 3C). Immunohistochemistry revealed expression of CD8 $\alpha$, CD4, CD11c, CD86, MHC II (I-A ${ }^{\text {b }}$ allele), and CD11b within periglomerular infiltrates (Figure 3, $\mathrm{D}-\mathrm{I}$ ), indicating the presence of $\mathrm{T}$ cells and antigen-presenting cells, such as DCs and macrophages.

DC subset changes in infiltrated kidneys. The expression of CD11c, MHC II, and CD86 within the infiltrate (Figure 3, F-H) suggested the presence of DCs. Indeed, flow cytometry revealed $\mathrm{CD} 11 \mathrm{c}^{+} \mathrm{MHC} \mathrm{II}^{+}$ cells that expressed CD11b either at high or intermediate levels (Figure 4A) but lacked CD8 and B220 (Supplemental Figure 5), identifying them as conventional CD8- DCs. DC numbers were higher in infiltrated kidneys, and this was mostly due to an increase in $\mathrm{CD} 11 \mathrm{c}^{+} \mathrm{CD} 11 \mathrm{~b}^{\text {hi }}$ cells, whereas CD $11 \mathrm{c}^{+} \mathrm{CD} 11 \mathrm{~b}^{\text {int }}$ cells hardly changed in number (Figure 4B), suggesting that the latter might represent resident kidney DCs and that the former may have been derived from circulating precursors, e.g., from monocytes, which express similarly high levels of CD11b (53). In support of this interpretation, the CD11bi cells expressed high levels of Gr1 (Figure $4 \mathrm{C}$ ), a marker of proinflammatory monocytes, which can give 
A

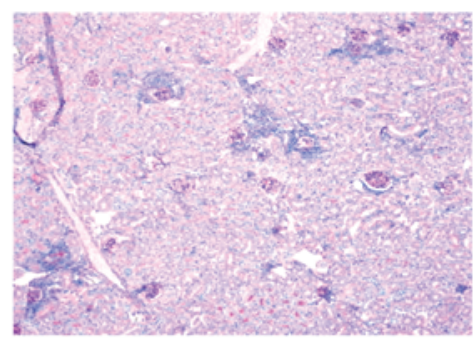

B

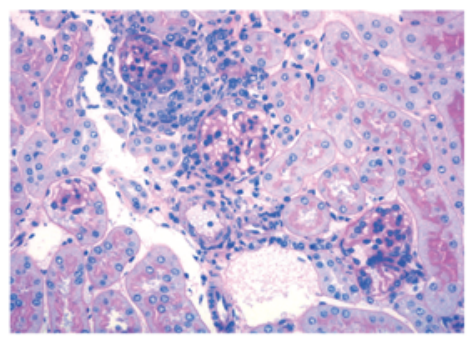

C
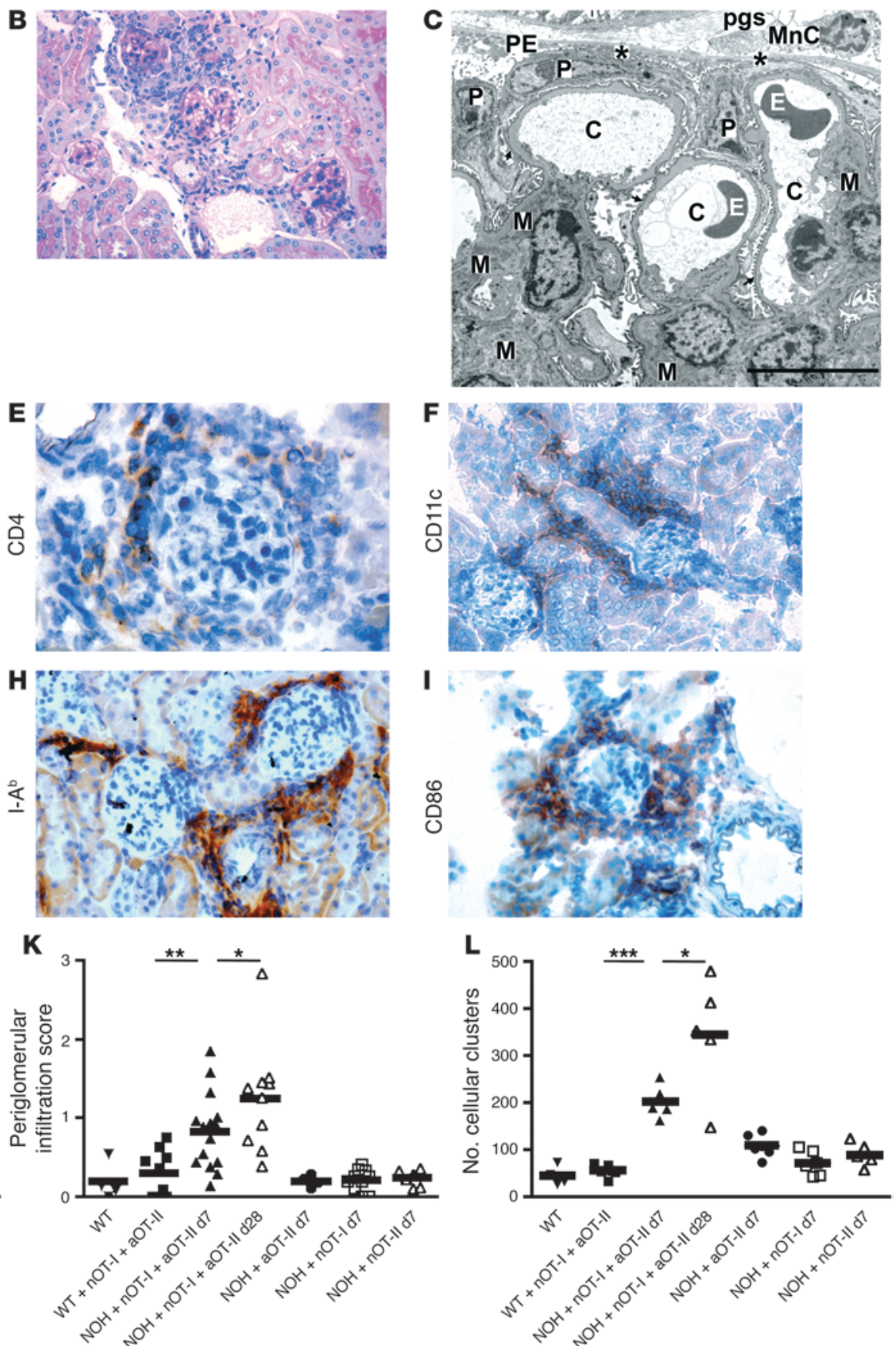

Figure 3

Histological analysis of periglomerular infiltrates in $\mathrm{NOH}$ mice. (A-C) PAS staining (A and $\mathbf{B})$ or electron microscopy analysis (C) of kidney sections of $\mathrm{NOH}$ mice injected with $5 \times 10^{6}$ OT-I cells and $5 \times 10^{6}$ activated OT-II cells 7 days before analysis. Note in C multiple contacts of podocytes with parietal epithelia separated by a very thin membrane from the periglomerular infiltrate. C, capillary; $\mathrm{E}$, erythrocyte; $\mathrm{M}$, mesangium; $\mathrm{MnC}$, mononuclear cell; P, podocyte; PE, parietal epithelium; pgs, periglomerular space; asterisk, capsule membrane. Original magnification, $\times 3,000$. Scale bar: $10 \mu \mathrm{m}$. (D-I) Representative immunohistochemistry for expression of CD8 (D), CD4 (E), CD11c (F), CD11b (G), MHC II (I-A $\left.{ }^{b}\right)$ $(\mathbf{H})$, and CD86 (I). (J) The frequency of glomeruli surrounded by mononuclear infiltrates was determined in HE-stained kidney sections of NOH or non-Tg mice injected with OT-I and/or activated OT-II cells as indicated. Shown are data from a group of mice that repetitively received T cell injections on days 7, 14, and 21. That group was analyzed on day 28 (histology in Figure 8). (K) Affected glomeruli were scored for the severity of periglomerular infiltrates. Results are representative of 4 experiments in groups of 3-5 mice. (L) Quantitative analysis of 2 of these experiments, an example of which was given in Supplemental Figure 7. In $\mathbf{J}-\mathbf{L}$, symbols indicate results from individual mice and the bars their mean. ${ }^{*} P<0.05 ;{ }^{* *} P<0.01 ;{ }^{* *} P<0.001$. 
A

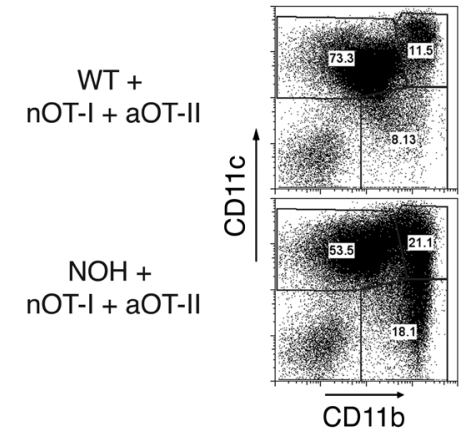

B

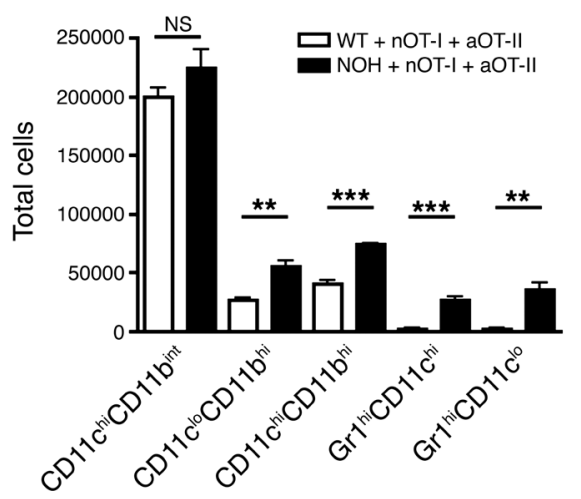

C

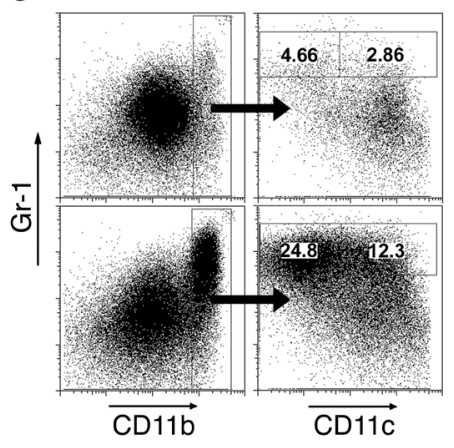

\section{Figure 4}

DC subsets in infiltrated kidneys. (A) MHC $\mathrm{Il}^{+}$cells in kidney single-cell suspensions from $\mathrm{NOH}$ (lower dot plot) or non-Tg (upper dot plot) mice injected with $5 \times 10^{6}$ OT-I cells and $5 \times 10^{6}$ activated OT-II cells were analyzed for expression of CD11b versus CD11c. (B) Absolute numbers of

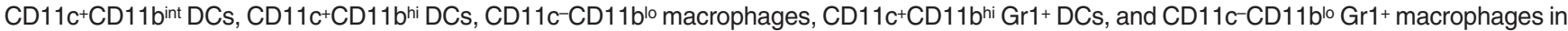
groups of 4 OT-I/II-injected NOH (black bars) or non-Tg (white bars) mice. (C) Dot plots shown as in A except that Gr1 was given instead of CD11c (left 2 dot plots). The right 2 dot plots show expression of CD11c versus Gr1 on the CD11 bi cells represented in A. Numbers in quadrants of dot plots indicate the proportion of cells. Results are representative of 3 experiments. ${ }^{* \star} P<0.01 ;{ }^{* \star} P<0.001$. Data are presented as mean \pm SD.

rise to both DCs and macrophages $(38,53,54)$. Further analysis of CD11b ${ }^{\text {hi }}$ cells confirmed Gr1 expression on both CD11b ${ }^{\text {hi }} \mathrm{CD} 11 \mathrm{c}^{+}$ and $\mathrm{CD} 11 \mathrm{~b}^{\text {hi }} \mathrm{CD} 11 \mathrm{c}^{-}$cells (Figure $4 \mathrm{C}$ ), consistent with recruitment of proinflammatory DCs and macrophages. Quantitative analysis verified that $C D 11 b^{\text {hi }} C D 11 c^{-}$macrophages had increased in number and that nearly all of them expressed Gr1 (Figure 4B). Also, the increase of CD $11 \mathrm{c}^{+}$DCs in infiltrated kidneys was mostly due to Gr1-expressing cells (Figure 4B).

$D C s$ are essential for periglomerular infiltration. To determine whether DCs were necessary for the periglomerular infiltrate, we injected OT cells into $\mathrm{NOH} \times \mathrm{CD} 11 \mathrm{c}$-DTR double-transgenic mice and depleted CD $11 c^{+}$cells by DT injection after 5 days, when infiltrates were established. This reduced kidney DC numbers by $85 \%-90 \%$ (Figure 5A). Histological analysis demonstrated that the periglomerular infiltrate had completely disappeared after 40 hours (Figure 5, B, F, and G; Supplemental Figure 6) but remained detectable in doubletransgenic mice that had not received DT (Figure 5, D, F, and G; Supplemental Figure 6), and in NOH single-transgenic mice, regardless of DT injection (Figure 5, C, E, F, and G; Supplemental Figure 6). We reasoned that such rapid resolution of the infiltrate was unlikely due to depletion of DCs in the renal LN because cessation of T cell priming in this node would not affect previously primed $\mathrm{T}$ cells in the kidney. Therefore we concluded that kidney DCs were necessary for maintenance of the periglomerular infiltrate.

Intrarenal DCs present CTL-released glomerular antigen only to $T h$ cells. When we examined activation of DCs in infiltrated kidneys of $\mathrm{NOH}$ mice, expression of CD86 and CD40 was higher compared to that of DCs in non-Tg controls (Figure 6, A and B), and a DC subset produced IL-12 (Figure 6C). Notably, these activation signs were also detectable albeit less pronounced when activated OT-II cells were injected alone but not when only activated OT-I cells were transferred (Figure 6, A-C), suggesting that interaction with Th cells alone was sufficient for DC maturation and that CTLs could further increase it.

To verify this interpretation, we injected $\mathrm{NOH}$ mice with activated OT-I cells in order to release glomerular antigen, isolated kidney DCs on the following day, and cocultured them with OT-II cells. Indeed, such DCs could stimulate IFN- $\gamma$ production from OT-II cells (Figure $6 \mathrm{D})$. IL-2 production was only marginally increased, and this was not significant (data not shown). Cytokine production was induced neither by DCs from OT-I cell-injected non-Tg mice nor by DCs from $\mathrm{NOH}$ mice that had not been injected with OT-I cells (Figure 6D), confirming that indeed CTL-released antigen was presented. DCs from the spleens of OT-I cell-injected NOH mice did not stimulate OT-II cells (Figure 6D), demonstrating that released glomerular antigen was not systemically available for presentation. These findings confirmed that intrarenal presentation of glomerular antigen by DCs elicited cytokine production by specific Th cells.

Interaction between kidney DCs and Th cells causes intrarenal CTL accumulation. We next studied the consequences of cytokine production for OT-I cells. Their numbers were increased in the infiltrated kidneys of $\mathrm{NOH}$ mice to more than 10 times the amount in the kidneys of non-Tg mice (Figure 7A), and they produced more IFN- $\gamma$, which indicated their activation (Figure 7B). Such an increase was not observed in the spleen (Figure 7C), demonstrating that OT-II cells caused local expansion of OT-I cells in the kidney. OT-I cell numbers in both kidney and spleen of $\mathrm{NOH}$ mice injected with OT-I cells alone were lower than in the respective organs of non-Tg mice (Figure 7, A and C), presumably indicating the beginning of their systemic deletion by cross-tolerance (40). Also, this reduction was prevented by OT-II cells (Figure 7C), demonstrating that these cells also exerted a distinct helper function, impairment of crosstolerance (40). The numbers of intrarenal OT-II cell numbers were not affected by coinjection of OT-I cells (Figure 7D), confirming that the former acted upstream of the latter. These findings indicated that interaction between kidney DCs and OT-II cells caused intrarenal accumulation of OT-I cells.

This increase of OT-I cells in the kidney did not result from their local proliferation because these cells did not express the Ki-67 proliferation marker when analyzed by intracellular flow cytometry (Figure 7E). To study the possibility of recruitment of OT-I cells, we determined expression of CCR5 ligands, which are produced by Th cells to attract CTLs (55). Indeed, we noted that OT-II cells isolated from infiltrated kidneys of $\mathrm{NOH}$ mice expressed 
A

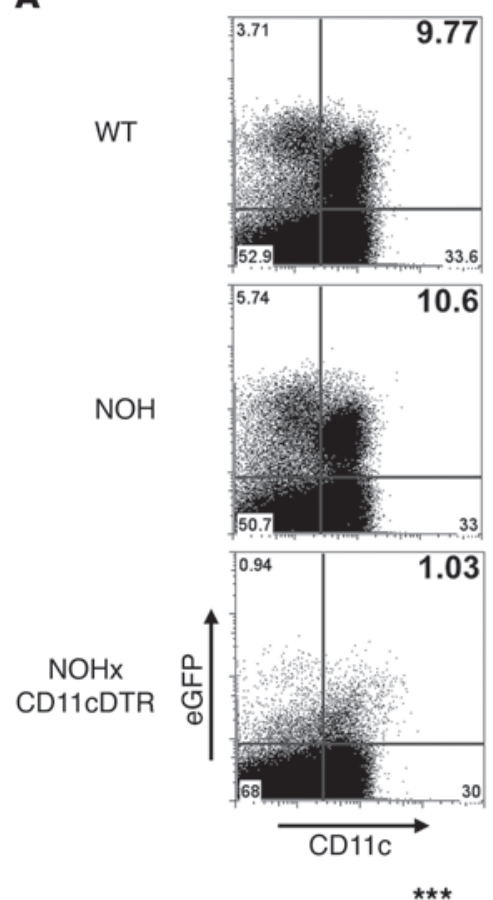

B $\mathrm{NOH} \times \mathrm{CD} 11 \mathrm{CDTR}+\mathrm{DT}$

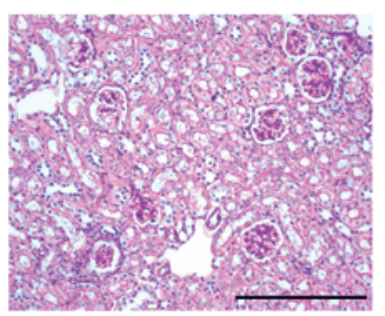

D $\mathrm{NOH} \times \mathrm{CD} 11 \mathrm{CDTR}+\mathrm{DT}$

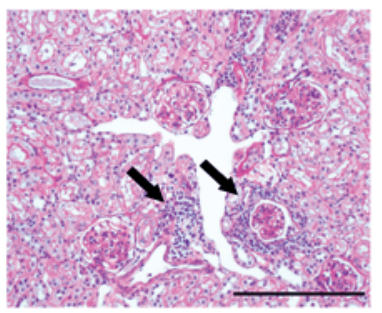

C

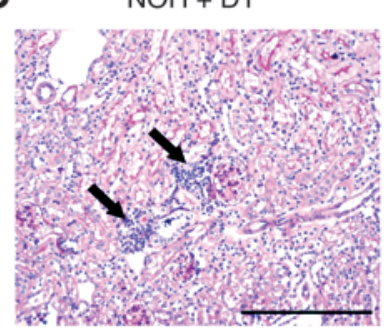

E

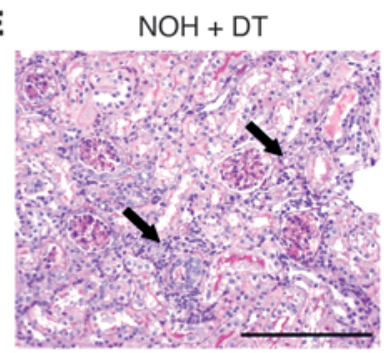

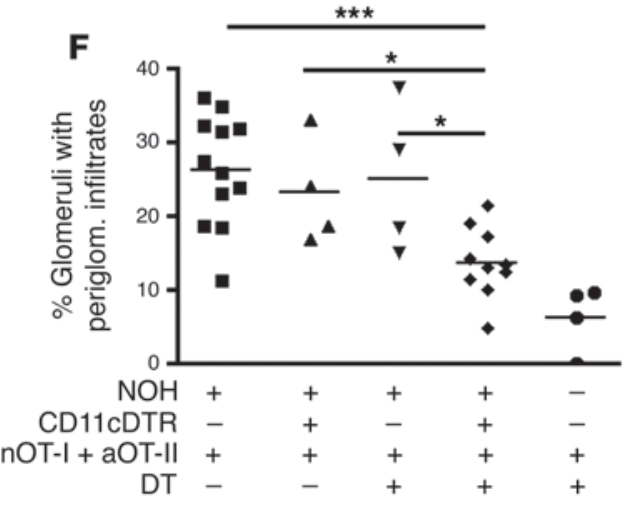

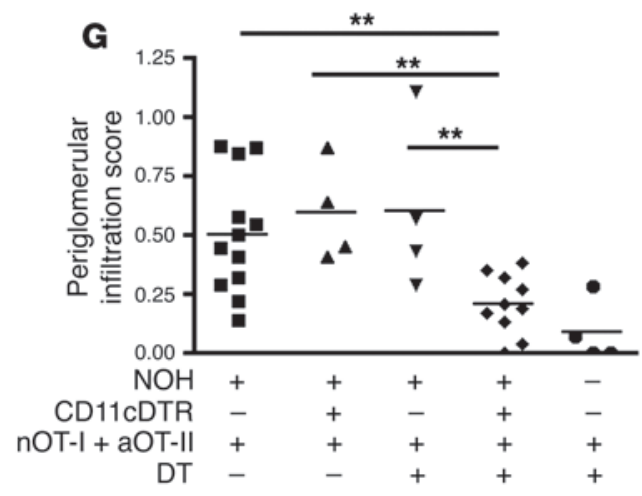

Figure 5

DCs are essential for periglomerular infiltration. (A) $4 \mathrm{ng} / \mathrm{g}$ body weight DT was injected into WT, NOH, or NOH $\times$ CD11c-DTR mice that had received $5 \times 10^{6}$ OT-I cells and $5 \times 10^{6}$ activated OT-II cells 5 days before. After an additional 40 hours, kidney single-cell suspensions were examined for surviving DCs by flow cytometry for CD11C+ and eGFP expression within the transgene. Dot plots show representative results, and a quantitative analysis is given in the same panel to the right. Numbers in quadrants of dot plots indicate the proportion of cells. (B-G) $5 \times 10^{6}$ OT-I cells and $5 \times 10^{6}$ activated OT-II cells were injected into $\mathrm{NOH} \times \mathrm{CD} 11 \mathrm{c}-\mathrm{DTR}$ (B and $\left.\mathbf{C}\right)$ or NOH mice (D and E). After 5 days, DT was injected (B and C). Kidney sections were scored after 40 hours for frequency $(\mathbf{F})$ and severity $(\mathbf{G})$ of infiltrates. Representative H\&E stainings are shown in B-E. Scale bars: $400 \mu \mathrm{m}$. Semiquantitative analyses show 2 further controls: non-Tg mice injected with DT and NOH mice not injected with DT. Symbols indicate sections from individual mice and the bars their mean. Results are representative of 2 experiments. ${ }^{\star} P<0.05$; ${ }^{\star \star} P<0.01$; ${ }^{* \star \star} P<0.001$. Data are presented as mean \pm SD.

mRNA levels of the CCR5 ligands CCL3, CCL4, and CCL5 that were $2.5,6$, or 2 times higher, respectively, than those of non- $\mathrm{Tg}$ mice. Of these, the increase in CCL4 was statistically significant. (Figure 7F). Intrarenal OT-I cells did not show such an increase but instead showed 6.5 times greater levels of CCR 5 itself (Figure 7G). Also, OT-II cells showed a moderate 2-fold CCR5 increase in infiltrated kidneys, but this was not statistically significant (Figure $7 F)$. When periglomerular infiltrates were excised by laser dissection microscopy and their mRNA was compared to that from corresponding tubulointerstitial areas of non- $\mathrm{Tg}$ mice, an even more striking increase of CCR5 ligands was observed, which reached 200-fold for CCL4 (Figure 7H). Here CCL3 and CCL5 were also significantly increased, implicating cells other than OT-I or OT-II cells as the source. These findings suggested that OT-II cells stimulated by kidney DCs in periglomerular infiltrates recruited OT-I cells to the kidney via CCR5 ligands.

Chronic Tcell-mediated damage causes structural and functional kidney damage. We finally examined the consequences of periglomerular infiltration at later time points. To this end, we repetitively injected NOH mice at weekly intervals with OT-I and activated OT-II cells because we speculated that the short life span of activated $\mathrm{T}$ cells might limit renal damage after a single injection. No pathology was seen when OT-I or OT-II cells were injected separately (data not shown). Coinjection resulted in pronounced tubulointerstitial 
A

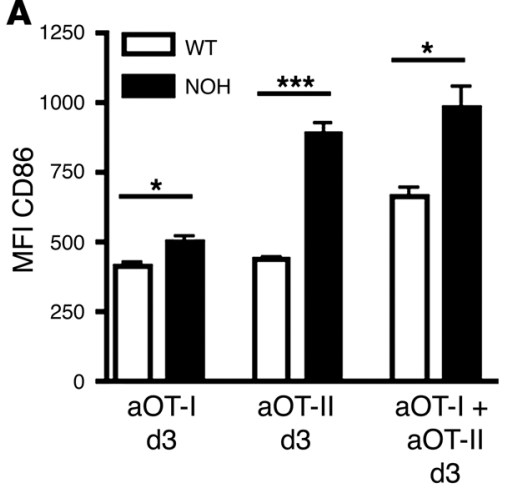

C

WT
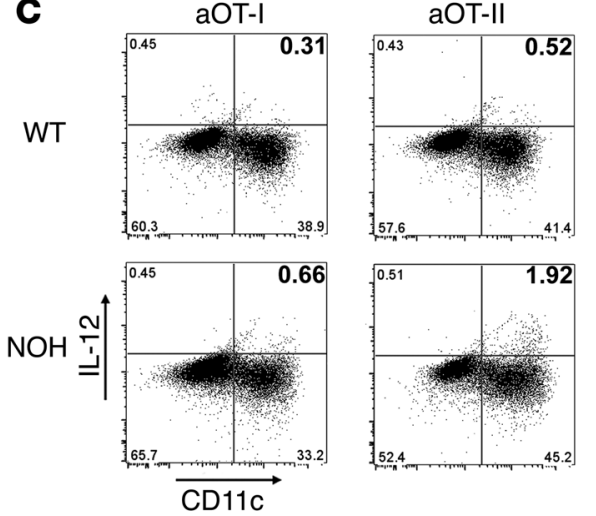

B

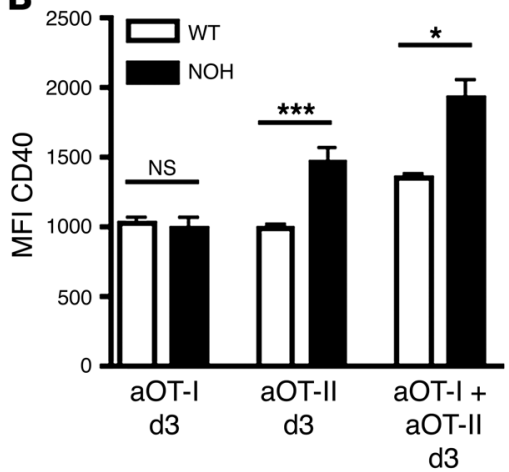

aOT-I + aOT-II D

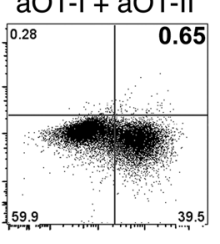

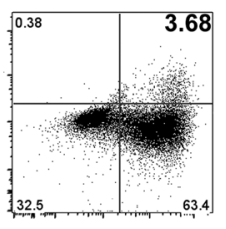

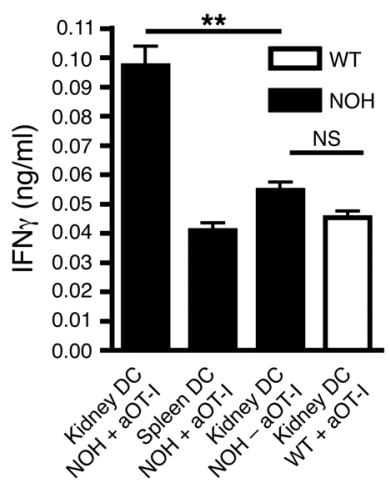

Figure 6

Kidney DCs present CTL-released glomerular antigen to OT-II cells. (A-C) DC maturation markers CD86 (A) and CD40 (B) and intracellular IL-12 production (C) were determined on $\mathrm{CD}_{11 \mathrm{C}^{+} \mathrm{MHC} \mathrm{II}}{ }^{+}$ kidney DCs on day 3 after injection of $5 \times 10^{6}$ activated OT-I cells and/or $5 \times 10^{6}$ activated OT-II cells as indicated. OT-I cells were used here also in an activated state to synchronize their effector phase with that of activated OT-II cells. Numbers in quadrants of dot plots indicate the proportion of cells. (D) $\mathrm{NOH}$ and non-Tg mice were injected with activated OT-I cells $(+\mathrm{aOT}-\mathrm{I})$ or not $(-\mathrm{aOT}-\mathrm{I})$. After 3 days, CD11 $\mathrm{C}^{+} \mathrm{DCs}$ were isolated from the kidney and spleen. $5 \times 10^{6}$ DCs were cultured with $5 \times 10^{6}$ OT-II cells. After 2 days, IFN- $\gamma$ concentrations in the supernatant were determined by ELISA. Results are representative of 2 experiments. ${ }^{*} P<0.05 ;{ }^{* *} P<0.01$; ${ }^{* \star *} P<0.001$. Data are presented as mean $\pm \mathrm{SD}$. infiltration by mononuclear cells, intratubular protein casts, focal tubular atrophy, and focal segmental glomerular sclerosis with retraction of the glomerular tuft to the vascular pole on day 28 (Figure 8, A and B). Type IV collagen staining on day 28 but not on day 7 revealed increased interstitial matrix deposition in the outer areas of the infiltrates, denoting induction of fibrosis at later time points (Figure 8, C and D). Together, these findings indicated chronic tubulointerstitial damage.

Notably, we regularly observed OVA-expressing cells in the Bowman capsule wall of infiltrated kidneys on day 28 (Figure 8F). The glomerular damage at this time point did not permit unequivocal classification of these cells. On day 7, such cells were rare and their OVA-staining intensity seemed lower (Figure 8E).

By electron microscopy, we determined that the parietal cells of the Bowman capsule appeared activated, with an increased number of organelles and edematous cytoplasm (Figure 8G). Glomerular capillaries showed regular endothelial cells, basement membranes, and podocytes. The Bowman capsule membrane was thickened. The composition of the periglomerular mononuclear infiltrate was similar to that seen on day 7 (Figure 3C), but the infiltrate was more pronounced and embedded in increased extracellular matrix (Figure 8G).

Consistent with glomerular sclerosis, daily excretion of albumin per creatinine, an indicator of glomerular damage, had increased substantially from day 7 to day 28 (Figure $8 \mathrm{H}$ ). No albuminuria was detected when OT-I or OT-II cells were injected alone or when non- $\mathrm{Tg}$ recipients were used (Figure $8 \mathrm{H}$ and data not shown). Renal insufficiency as determined by creatinine clearance did not develop until day 28 (data not shown). Gel electrophoresis revealed that excreted protein was primarily of albumin and not of Ig size (Figure 8I), indicative of selective glomerular proteinuria. Very low albuminuria was found when activated OT-I cells were injected alone (Figure 8I), supporting the conclusion that $\mathrm{CD}^{+} \mathrm{T}$ cells could cause kidney damage but that the presence of Th cells was required for progression to manifest immunopathology and functional damage.

\section{Discussion}

The role of T cells in GN is controversial (2, 19-22, 25-28). Using the transgenic NOH model, we demonstrated that CTLs and Th cells specific for glomerular autoantigen can jointly induce periglomerular mononuclear infiltration that can progress to structural and functional kidney damage. Our analysis supports the following sequence of events: DCs in the renal LNs constitutively cross-presented glomerular antigen to specific CTLs by DCs in the renal LNs. Activated CTLs entered the kidney and released more glomerular antigen, which augmented cross-presentation in the renal LNs. Simultaneously, such antigen was taken up also by DCs of a different subtype in the kidney tubulointerstitium, which presented it to specific Th cells, resulting in activation of kidney DCs and Th cells and in intrarenal production of proinflammatory cytokines and chemokines. In response, further CTLs were recruited to the kidney as well as monocyte-derived proinflammatory DCs and macrophages. These immune effector cells formed and maintained periglomerular infiltrates and thereby contributed to immunopathology.

The present study shows that renal LN DCs not only cross-present tubular autoantigen (33) and innocuous small molecular weight antigen filtrated in the glomerulus (56) but also crosspresent glomerular autoantigen and induce CTL cross-tolerance. 


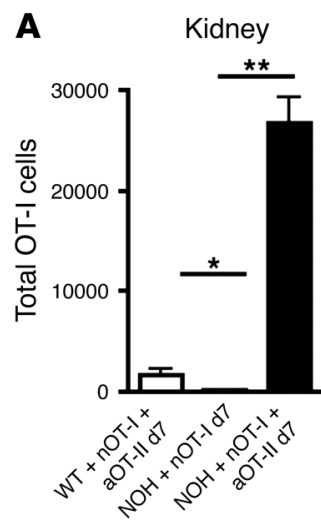

E

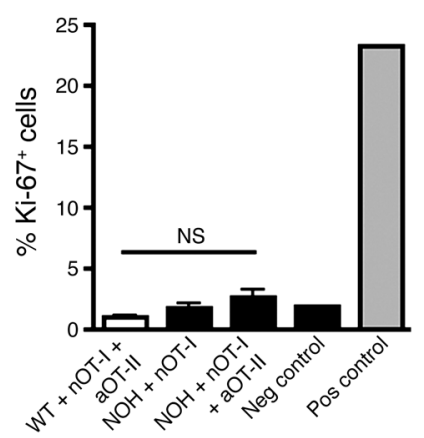

B

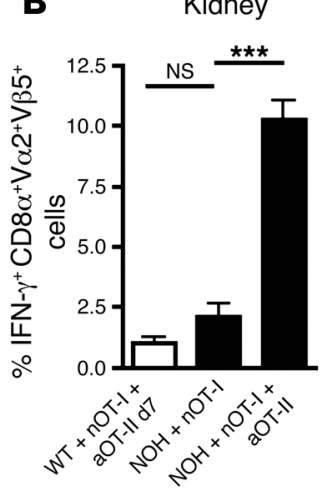

F

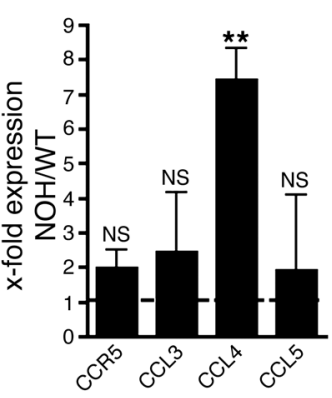

c

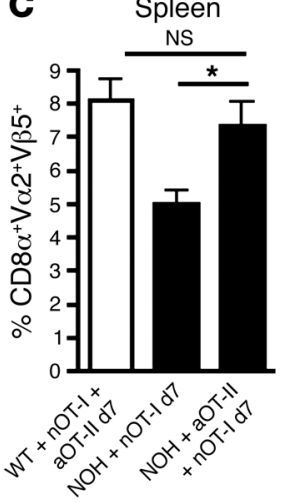

G

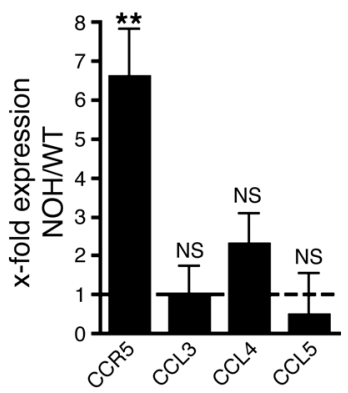

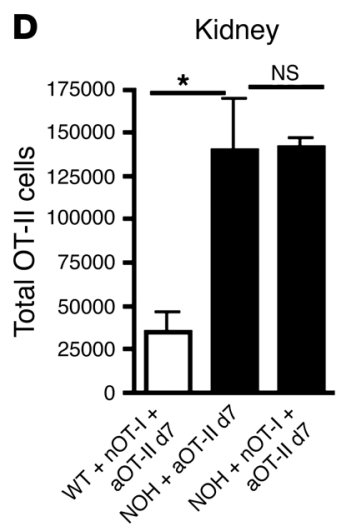

H

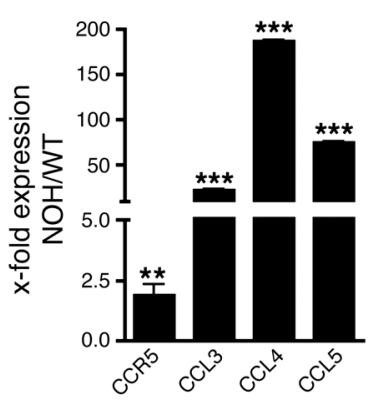

Figure 7

Interaction between kidney DCs and Th cells causes intrarenal CTL accumulation. NOH (black bars) or non-Tg (white bars) mice were injected with $5 \times 10^{6}$ OT-I cells alone or together with $5 \times 10^{6}$ activated OT-Il cells. (A-C) After 7 days, single-cell suspensions of the kidney (A and B) or the spleen (C) were analyzed by flow cytometry to determine numbers of $C D 8^{+} \mathrm{V} \alpha 2^{+} \mathrm{V} \beta 5^{+} \mathrm{OT}-\mathrm{I}(\mathbf{A}$ and $\mathbf{C})$ or proportions of $(\mathbf{B})$ IFN- $\gamma-$ producing OT-I cells. (D) Numbers of CD4+ $4^{+} 2^{+} \mathrm{V} \beta 5^{+}$OT-II cells were determined after injection of $5 \times 10^{6}$ OT-II cells alone or together with $5 \times 10^{6}$ activated OT-II cells. (E) Experiments depicted in A-C show determination of Ki-67+ OT-I cells. In vitro-activated OT-II cells served as positive control, naive OT-II cells as negative control for proliferating cells. ( $F$ and $\mathbf{G}) \mathrm{V} \alpha 2^{+} \mathrm{V} \beta 5^{+} \mathrm{CD} 4^{+} \mathrm{OT}-\mathrm{II}(\mathbf{F})$ or $\mathrm{V} \alpha 2^{+} \mathrm{V} \beta 5^{+} \mathrm{CD} 8^{+} \mathrm{OT}-\mathrm{I}$ cells $(\mathbf{G})$ were sorted from kidney cell suspensions of experiments depicted in A-C. mRNA encoding CCL3, CCL4, CCL5, and CCR5 was determined, and the ratio between cells from $\mathrm{NOH}$ and non-Tg controls was displayed. $(\mathbf{H})$ Mononuclear cell infiltrates and noninfiltrated tubulointerstitial control areas were excised from kidney cryosections as shown in Supplemental Figure 8. mRNA encoding CCL3, CCL4, CCL5, and CCR5 was determined, and the ratio between infiltrates from injected $\mathrm{NOH}$ and non-Tg controls was displayed. Results are representative of 2 experiments. ${ }^{*} P<0.05$; ${ }^{\star \star} P<0.01 ;{ }^{* \star} P<0.001$. Data are presented as mean \pm SD.

Such tolerance may explain the minor kidney damage that CTLs induced without Th cells, which was evident solely by the release of some podocyte antigen. It has been previously shown that CTLs activated without help are functionally deficient and doomed to die $(40,43,57)$, and this may apply also to glomerular antigen-specific CTLs. Formal demonstration of their intrarenal death, however, was impossible because we could hardly detect any CTLs in the kidney when autoreactive Th cells were lacking. Provision of such helper cells rescued autoreactive CTLs in our system not only by preventing cross-tolerance but also by an additional mechanism that operated within the kidney (40), which resulted in their intrarenal accumulation and immunopathology. CTLs rescue critically depended on DCs, since their depletion dissolved the periglomerular infiltrate within less than 2 days. Such rapid resolution cannot be explained by abrogated $\mathrm{T}$ cell activation in the renal LNs. We therefore concluded that kidney DCs were essential for maintenance of the periglomerular infiltrate, implying a role for DCs in the progression of kidney disease. Ostensibly, this seemed to contradict our previous finding that DC depletion aggravated nephrotoxic nephritis (18). However, in that study, all DCs were depleted at an early point of nephritis (day 4) and therefore the resident DCs were also removed. It is possible that only these were responsible for the protective effect. This would be consistent with recent evidence that resident and recruited DC subsets derive from distinct precursors and perform distinct functions $(38,39,53,54,58)$. Thus, it is possible that the recruited $\mathrm{Gr} 1^{+}$DC subset sustained the periglomerular infiltrate.

An advantage of the $\mathrm{NOH}$ model over transgenic models with antigen expression in other organs, in particular pancreatic islets that are rapidly destroyed by CTLs (40), is the persistence of the mononuclear infiltrate over several days, which allowed us to dissect the crosstalk between the participating immune cells. We found that renal DCs presented CTL-released glomerular antigen only to Th cells. This was consistent with the reported inability of CD8conventional DCs (which include kidney DCs; ref. 10) to cross-present antigen (34-39) and implied their inability to directly interact with OT-I cells in an MHC I-restricted manner. Moreover, kidney DCs do not express the mannose receptor (56), which is required for uptake of OVA for cross-presentation (37). Finally, if kidney DCs could cross-present, then they theoretically should become sus- 
A

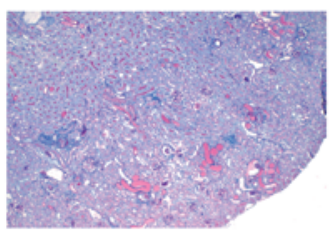

B

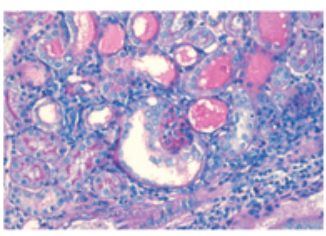

c

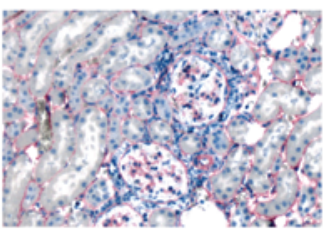

D

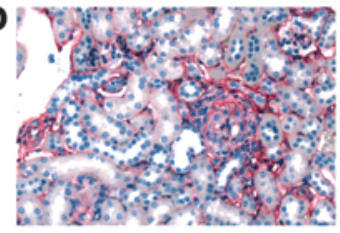

E
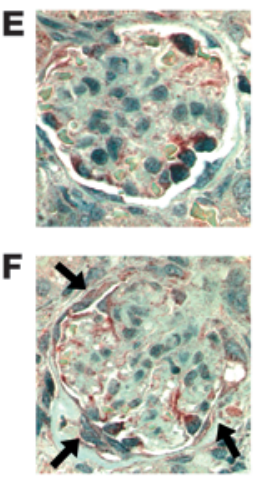

G

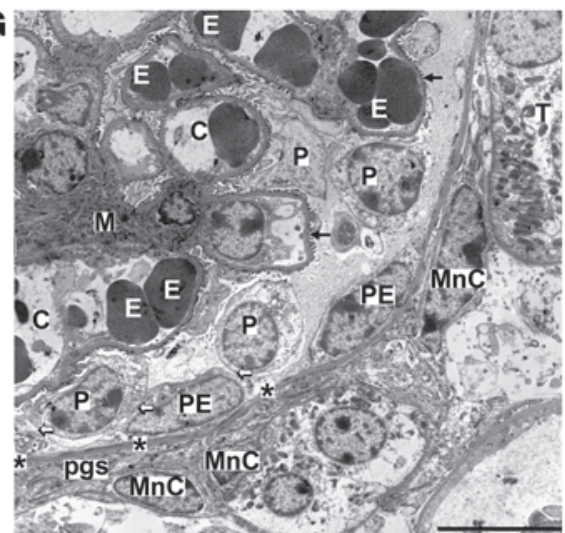

\section{H}

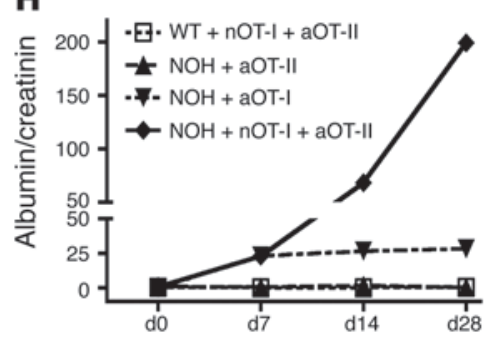

I

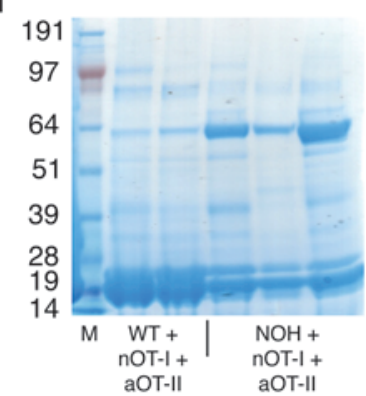

\section{Figure 8}

Repetitive OT cell injection causes functional and structural kidney damage in NOH mice. NOH mice were injected with $5 \times 10^{6}$ OT-I cells and with $5 \times 10^{6}$ activated OT-II cells on days $0,7,14$, and 21 . On day 7 ( $\mathbf{C}$ and $\left.\mathbf{E}\right)$ or day 28 (A, B, D, and $\left.\mathbf{F}-\mathbf{I}\right)$, kidneys were taken for PAS staining (A and B), type IV collagen staining for fibrotic areas (C and D), OVA staining (E and F; black arrows indicate OVA ${ }^{+}$cells in Bowman capsule wall), and electron microscopy (G). White arrow, contact between podocytes and parietal cells; black arrow, podocyte foot processes. Original magnification, $\times 3,000$. Scale bar: $10 \mu \mathrm{m}$. (H) Daily excretion of albumin $(\mathrm{g} / \mathrm{l})$ per creatinine $(\mathrm{g} / \mathrm{l})$ was determined in overnight urine of groups of $\mathrm{NOH}$ or non-Tg mice injected with nOT-I and/or activated OT-Il cells. (I) $20 \mu$ urine from mice in groups denoted by diamonds and open squares on day 7 were separated by gel electrophoresis and stained with Coomassie blue. Results are representative of 2 experiments. ${ }^{\star} P<0.05 ;{ }^{\star \star} P<0.01 ;{ }^{* \star \star} P<0.001$.

ceptible to CTL-mediated lysis and their numbers should decline rather than accumulate, as observed here. Thus, CTL rescue most likely was mediated by soluble mediators produced in response to intrarenal Th cell-DC crosstalk, i.e., IFN- $\gamma$ by Th cells and/or IL-12 by DCs, which are known to stimulate CTLs and to aggravate some forms of nephritis $(26,27,59)$. Although Th cell-derived IFN- $\gamma$ possibly also activated intrarenal macrophages to contribute to immunopathology in a classical DTH-like manner, CTLs were essential in our system because injection of Th cells alone did not cause immunopathology. CTL accumulation did not result from their intrarenal proliferation. Instead, we found evidence for recruitment to the kidney by Th cell-produced CCR5 ligands. CTL recruitment is consistent with previous studies on primary interstitial nephritis, which concluded that Th cells specific for tubular antigen permitted access of specific circulating CTLs to the tubulointerstitium by unknown mechanisms (29-31). It is also consistent with the recent demonstration that Th cells recruit naive CTLs to the DCs for priming in secondary lymphatics via CCR5 ligands (55). Our results suggest that these ligands also attract effector CTLs to nonlymphoid tissue, here the kidney. In support of this notion, nearly all kidney-infiltrating CTLs in experimental nephritis models have previously been reported to express CCR5 (60).

Although our findings clearly demonstrate that the periglomerular infiltrate was maintained by antigen and by DCs presenting it, the mechanism by which periglomerular DCs could physically acquire podocyte antigen remained unclear. Migratory DCs are unlikely to transport antigen because they are normally absent from glomeruli (10) and were not detected there in the present study. We found no evidence for transgene expression in parietal epithelium in healthy mice, and this has not been reported in other transgenic mice harboring the human nephrin promoter used here (48). Thus, podocyte antigen may be shed, reabsorbed from the glomerular filtrate, and acquired by tubulointerstitial DCs. In support of this hypothetical mechanism, we have recently observed that kidney DCs can take up filtrated antigen from the glomerular ultrafiltrate (56). When inflammation is established, the proximity between podocytes and parietal cells and the very thin basement membrane that separates the periglomerular infiltrate may facilitate antigen transfer. Alternatively, podocytes may relocate to the Bowman capsule, as recently described in experimental crescentic nephritis (61), or inflamed parietal cells themselves may acquire podocyte-like properties. Indeed, on day 28 , we observed OVA-expressing cells in the Bowman capsule wall adjacent to the periglomerular infiltrates and DCs. Future studies may clarify whether these cells represented relocated podocytes or parietal cells that expressed antigen driven by the nephrin promoter under inflammatory conditions.

Transgenic models such as that studied here generally use high numbers of autoreactive T cells (40, 45-47), which does not resemble clinical situations and therefore warrants caution in extrapolating results to human disease. Nevertheless, the requirement 
of very high numbers of autoreactive Th cells and CTLs, which needed to be stimulated by distinct DCs in different locations to induce immunopathology, indicated that several checkpoints exist to avoid T cell-mediated kidney disease. Furthermore, our study revealed the form of immunopathology resulting from $T$ cells specific for glomerular antigen. Such T cells caused periglomerular mononuclear infiltration and parietal epithelial cell inflammation but not typical signs of direct podocyte injury such as foot-process fusion. Although this morphology is not identical to that of the classical types of human GN, periglomerular infiltration is frequently observed in T cell-dependent experimental GN models, e.g., lupus or crescentic nephritis $(10,11)$, and resembles lesions usually seen at the margins of focal segmental and necrotizing lesions of pauci-immune complex GN $(5,62)$. The detection and immunohistological characterization of periglomerular infiltrates with respect to proinflammatory DC subsets may be of diagnostic value in glomerular diseases, especially when the use of T cell-suppressive drugs is considered.

In summary, our findings demonstrate that a joint attack of glomerular antigen-specific CTLs and Th cells can lead to distinct immunopathology predominantly affecting the tubulointerstitium. These findings may provide the pathophysiologic basis for the long-known fact that kidney function and its prognosis are closely correlated with tubulointerstitial and not with glomerular damage, even in primary glomerular disease $(1,5)$. Presentation of glomerular antigen by activated or recruited kidney DCs may represent a previously unrecognized mechanism by which glomerular injury may spread to the tubulointerstitium and sustain inflammation there. This theory suggests that targeting proinflammatory DCs or their blood precursors represents what we believe to be a novel therapeutic option to delay or even stop kidney disease progression.

\section{Methods}

Mice and reagents. WT, NOH, OT-I Rag-/-, OT-II, and CD11c-DTR/GFP mice on the $\mathrm{C} 57 \mathrm{BL} / 6$ background were bred and maintained in the animal facilities of the University of Bonn (House of Experimental Therapy [HET]) under specific pathogen-free conditions and used for experiments at between 8 and 16 weeks of age. All studies were reviewed and approved by an external review board (Bezirksregierung Köln, Cologne, Germany). DCs were depleted in CD11c-DTR/GFP mice by injecting $4 \mathrm{ng} / \mathrm{g}$ body weight DT i.p. All reagents, if not otherwise specified, were from Sigma-Aldrich.

Generation of transgenic NOH mice. The 715-bp XhoI-HindIII fragment containing the rat insulin promoter was excised from the POVA/HEL plasmid used to generate transgenic RIP-mOVA/HEL (where $m$ stands for membrane bound) (ROH) mice (49), which contained cDNA for the human transferrin receptor membrane domain for the OVA (aa 161-407), the HEL gene, and an SV40 polyA tail. It was replaced with the human nephrin promoter, which had been amplified from the plasmid nephrin/PCR2.1 (48) using the primers GACAGAGGTGGGAGAATTACTT and ACAGCGCCCGCTGCCAGC and was then ligated into the blunted $5.7-\mathrm{kb}$ pOVA/HEL fragment. A 3.79-kb PvuI-BamHI fragment was cut out and injected into pronuclei of fertilized C57BL/6 oocytes. Two founder lines were generated, both of which showed similar immunopathology in our system. The correct nucleotide sequence of the transgene was verified by sequencing.

Cell transfer experiments. OT-I and OT-II cells were prepared from the spleen and LNs of transgenic mice as described and labeled using CFSE (Molecular Probes; Invitrogen) as described (40). OT-I and OT-II cells were activated by coculture with irradiated OVA peptide-loaded splenocytes for 4 days. Mice were injected with $5 \times 10^{6} \mathrm{~T}$ cells.
Isolation of primary DCs and T cells. Kidneys were injected with RPMI 1640 containing $1 \mathrm{mg} / \mathrm{ml}$ collagenase D (Roche) and $0.2 \mathrm{mg} / \mathrm{ml}$ DNAse I. Undigested tissue fragments and kidney tubular cells were removed by sedimentation as described (18). For ex vivo cocultures, DCs and T cells were purified using nanobead-labeled antibodies (Miltenyi Biotec). Magnetic bead separation was done according to the manufacturer's instructions. Purity was usually $80 \%$ to $90 \%$.

Flow cytometry. The following mAbs from eBioscience were used: CD4 (clone GK1.5), CD8a (53-6.7), CD11c (HL3), I-Ab (AF6-120.1), CD11b

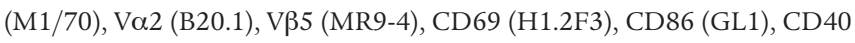
(3/23), IL-12p40 (C17.8), and IFN- $\gamma$ (XMG1.2). Ki-67 (SP6) was a gift from Thermo Scientific. For intracellular staining, cells were cultured for 5 hours with $1 \mu \mathrm{l} / \mathrm{ml}$ GolgiPlug (BD Biosciences - Pharmingen), treated with 24G2 hybridoma supernatant to block Fc receptors, stained for surface markers, fixed with $2 \%$ PFA, and permeabilized with BD Perm/Wash. Absolute cell numbers were determined by adding fixed numbers of Calibrite APC beads (BD) before measurement as internal reference. Dead cells were excluded with Hoechst 33342. Flow cytometry was performed on a FACSCanto II (BD) and data analysis with FlowJo software (Tristar). Cell sorting was performed on a FACSVantage (BD).

Histology. Tissues were fixed in 4\% PFA overnight, and embedded in paraffin. 5 - $\mu \mathrm{m}$ sections were stained with PAS or Sirius red using standard techniques. Extent of mononuclear infiltration was semiquantitatively determined in PAS-stained sections using the following score: 0 , no infiltration; 0.5 , periglomerular infiltrates discontinuous or less than 3 layers; 1 , continuous periglomerular infiltration with at least 2 cell layers; 2 , severe infiltration with more than 4 layers; and 3, severe infiltration plus crescents of Bowman capsule or glomerular sclerotic lesions. A minimum of 50 glomeruli per section was counted. Quantitative analysis of infiltrates was performed using SCAN-R software (Olympus): 1 to 2 independent areas of 16 single images $(434 \times 330 \mu \mathrm{m})$ per section of 3 to 4 animals per group were analyzed for clusters of more than 3 cells showing a lymphocyte size threshold (example shown in Supplemental Figure 7).

For immunohistochemistry, 5- to 7- $\mu \mathrm{m}$ acetone-fixed cryosections were stained with biotinylated $\mathrm{mAb}$, followed by ABC-SP Kit (Vector Laboratories) and diaminobenzidine and hematoxylin counterstaining. For type IV collagen staining, paraffin sections were treated with protease 24 , washed, blocked with horse serum, incubated with goat anti-type IV Collagen (SouthernBiotech), blocked again overnight, incubated with biotinylated donkey anti-goat Ig, revealed with streptavidin-AP, developed with new fuchsin, and counterstained with hemalaun. For OVA immunohistochemistry, kidneys were fixed in $4 \%$ formalin overnight, and $1-\mu \mathrm{m}$ paraffin sections were cut. Antigen was retrieved with $5 \mathrm{mg} / \mathrm{ml}$ proteinase type XXIV for 15 minutes at $37^{\circ} \mathrm{C}$. After a 30 -minute block in $5 \%$ goat serum (Vector Laboratories), sections were incubated with homemade polyclonal rabbit anti-OVA Ab (1:500 in 5\% goat serum) overnight at $4{ }^{\circ} \mathrm{C}$. Binding was visualized by biotinylated goat anti-rabbit $\mathrm{Ab}$ (Vector Laboratories) diluted 1:200 in 5\% goat serum for 30 minutes, and revealed by ABC-AP Kit (Vector Laboratories) followed by new fuchsin (Merck) and hematoxylin counterstaining, and analysis using an Axioskop (Zeiss). Electron microscopy was performed on a Zeiss EM900. Images were generated by multiple image alignment of 16 individual images.

Laser microdissection. 8- $\mu \mathrm{m}$ cryosections of mouse kidneys were stained for alcohol-based cresyl violet acetate by air drying sections for 1 minute, incubated for 2 minutes in precooled 75\% EtOH, and dipped for $20 \mathrm{sec}$ onds in $1 \%$ cresyl violet acetate dissolved in EtOH. Then slides were washed in $75 \%$ and $100 \% \mathrm{EtOH}$ for 30 seconds each and air dried for 10 minutes. Tissue was excised using a PALM MicroBeam IP 230V Z microscope for laser pressure catapulting (P.A.L.M. Microlaser Technologies) as described previously (63) and in Supplemental Figure 8. RNA from microdissected tissue was prepared using the PALM RNA extraction kit. 
Real-time PCR. RNA was isolated using the RNeasy Micro Kit (QIAGEN). cDNA was synthesized using random hexamer primers. RT-PCR was performed on an ABI Prism 7000 Sequence Detection System (Applied Biosystems) using the following settings: 40 cycles of 15 seconds denaturation at $95^{\circ} \mathrm{C}$, and 1 minute primer annealing and elongation at $60^{\circ} \mathrm{C}$ as described (63). $1.5 \mu \mathrm{l}$ cDNA was used with $2.5 \mu \mathrm{l}(0.9 \mu \mathrm{M})$ specific primers and $12.5 \mu \mathrm{l}$ of $2 \times$ Platinum SYBR Green qPCR SuperMix (Invitrogen). All samples were run in duplicates and normalized to $18 \mathrm{~S}$ rRNA.

Miscellaneous measurements and statistical analysis. IFN- $\gamma$ was measured by ELISA (R\&D Systems). Proteinuria was quantified using metabolic cages. To guarantee sufficient quantities of urine, all mice in a group were placed overnight in the same cage. Urinary albumin was determined by a commercial ELISA kit (Bethyl Laboratories Inc.); creatinine measurements and SDS-PAGE ( $5 \%$ acrylamide gel) were carried out by standard methodology. Two-tailed Student's $t$ test analysis was done using Prism software (GraphPad Software). RT-PCR data were analyzed by comparing experimental groups by Kruskal-Wallis test with post hoc analysis by Mann-Whitney test. Results were given as mean \pm SD. $P<0.05$ was considered significant.

\section{Acknowledgments}

We thank Booshini Fernando, Inge Heim, and Anett Peters for technical assistance; Sjef Verbeek for microinjections; Thomas Zillinger and Jan-Eric Turner for help with quantitative RT-PCR; and Thermo
Scientific for a generous gift of Ki-67 (SP6) antibody. We acknowledge support by the Central Animal Facilities (House of Experimental Therapy [HET]) and the Flow Cytometry Core Facility of the Medical Faculty of the University of Bonn. F. Heymann was supported by a PhD fellowship from the German National Academic Foundation (Studienstiftung des deutschen Volkes) and by a BONFOR institutional grant. C.Kurts was supported by a career development grant from the German state of North Rhine-Westphalia and by grants from the SFB (Sonderforschungsbereich) TR57, SFB704 and a Heisenberg fellowship from the Deutsche Forschungsgemeinschaft (DFG). J. Floege received a further grant from the SFB TR57 of the DFG. U. Panzer was supported by DFG grant PA 754/6-3.

Received for publication December 22, 2008, and accepted in revised form March 25, 2009.

Address correspondence to: Christian Kurts, Institute of Experimental Immunology, IMMEI, Universitätsklinik Bonn, Sigmund-Freud-Str. 25, 53105 Bonn, Germany. Phone: 49-228-287-11031; Fax: 49-228287-11052; E-mail: ckurts@web.de.

Felix Heymann's present address is: Medical Clinic III, RWTH University of Aachen, Aachen, German.
1. Bohle, A., Strutz, F., and Muller, G.A. 1994. On the pathogenesis of chronic renal failure in primary glomerulopathies: a view from the interstitium. Exp. Nephrol. 2:205-210.

2. Nikolic-Paterson, D.J., and Atkins, R.C. 2001 The role of macrophages in glomerulonephritis. Nephrol. Dial. Transplant. 16(Suppl. 5):3-7.

3. Kriz, W., and LeHir, M. 2005. Pathways to nephron loss starting from glomerular diseases-insights from animal models. Kidney Int. 67:404-419.

4. Abbate, M., Zoja, C., and Remuzzi, G. 2006. How does proteinuria cause progressive renal damage? J. Am. Soc. Nephrol. 17:2974-2984.

5. Markovic-Lipkovski, J., Muller, C.A., Risler, T. Bohle, A., and Muller, G.A. 1990. Association of glomerular and interstitial mononuclear leukocytes with different forms of glomerulonephritis. Nephrol. Dial. Transplant. 5:10-17.

6. Foster, M.H. 2007. T cells and B cells in lupus nephritis. Semin. Nephrol. 27:47-58.

7. Couzi, L., et al. 2007. Predominance of CD8+ T lymphocytes among periglomerular infiltrating cells and link to the prognosis of class III and class IV lupus nephritis. Arthritis Rheum. 56:2362-2370.

8. Falk, M.C., et al. 1995. Infiltration of the kidney by alpha beta and gamma delta T cells: effect on progression in IgA nephropathy. Kidney Int. 47:177-185.

9. Cunningham, M.A., Huang, X.R., Dowling, J.P., Tipping, P.G., and Holdsworth, S.R. 1999. Prominence of cell-mediated immunity effectors in "pauci-immune" glomerulonephritis. J. Am. Soc. Nephrol. 10:499-506.

10. Kruger, T., et al. 2004. Identification and functional characterization of dendritic cells in the healthy murine kidney and in experimental glomerulonephritis. J. Am. Soc. Nephrol. 15:613-621.

11. Fujinaka, H., et al. 2007. Periglomerular accumulation of dendritic cells in rat crescentic glomerulonephritis. J. Nephrol. 20:357-363.

12. Duffield, J.S., et al. 2005. Conditional ablation of macrophages halts progression of crescentic glomerulonephritis. Am. J. Pathol. 167:1207-1219.

13. Kaissling, B., and Le Hir, M. 1994. Characterization and distribution of interstitial cell types in the renal cortex of rats. Kidney Int. 45:709-720.

14. Soos, T., et al. 2006. CX3CR1+ interstitial dendritic cells form a contiguous network throughout the entire kidney. Kidney Int. 70:591-596.

15. Woltman, A.M., et al. 2007. Quantification of dendritic cell subsets in human renal tissue under normal and pathological conditions. Kidney Int. 71:1001-1008.

16. Dong, X., et al. 2007. Resident dendritic cells are the predominant TNF-secreting cell in early renal ischemia-reperfusion injury. Kidney Int. 71:619-628.

17. Dong, X., Bachman, L.A., Miller, M.N., Nath, K.A., and Griffin, M.D. 2008. Dendritic cells facilitate accumulation of IL-17 T cells in the kidney following acute renal obstruction. Kidney Int. 74:1294-1309.

18. Scholz, J., et al. 2008. Renal dendritic cells stimulate IL-10 production and attenuate nephrotoxic nephritis. J. Am. Soc. Nephrol. 19:527-537.

19. Dixon, F.J. 1970. What are sensitized cells doing in glomerulonephritis? N. Engl. J. Med. 283:536-537.

20. Couser, W.G. 1999. Sensitized cells come of age: a new era in renal immunology with important therapeutic implications. J. Am. Soc. Nephrol. 10:664-665.

21. Bolton, W.K. 2002. What sensitized cells just might be doing in glomerulonephritis. J. Clin. Invest. 109:713-714.

22. Falk, R.J., and Jennette, J.C. 2002. ANCA are pathogenic - oh yes they are! J. Am. Soc. Nephrol. 13:1977-1979.

23. Stilmant, M.M., Bolton, W.K., Sturgill, B.C., Schmitt, G.W., and Couser, W.G. 1979. Crescentic glomerulonephritis without immune deposits: clinicopathologic features. Kidney Int. 15:184-195.

24. Bolton, W.K., et al. 1988. Transfer of experimental glomerulonephritis in chickens by mononuclear cells. Kidney Int. 34:598-610.

25. Neale, T.J., Tipping, P.G., Carson, S.D., and Holdsworth, S.R. 1988. Participation of cell-mediated immunity in deposition of fibrin in glomerulonephritis. Lancet. 2:421-424.

26. Kalluri, R., Danoff, T.M., Okada, H., and Neilson, E.G. 1997. Susceptibility to anti-glomerular basement membrane disease and Goodpasture syndrome is linked to MHC class II genes and the emergence of $\mathrm{T}$ cell-mediated immunity in mice. J. Clin. Invest. 100:2263-2275.

27. Tipping, P.G., and Holdsworth, S.R. 2006. T cells in crescentic glomerulonephritis. J. Am. Soc. Nephrol.
17:1253-1263.

28. Kurts, C., Heymann, F., Lukacs-Kornek, V., Boor, P., and Floege, J. 2007. Role of T cells and dendritic cells in glomerular immunopathology. Semin. Immunopathol. 29:317-335.

29. Mann, R., et al. 1985. Murine interstitial nephritis. IV. Long-term cultured L3T4+ T cell lines transfer delayed expression of disease as I-A-restricted inducers of the effector $\mathrm{T}$ cell repertoire. J. Immunol. 135:286-293

30. Meyers, C.M., and Kelly, C.J. 1991. Effector mechanisms in organ-specific autoimmunity. I. Characterization of a CD8 $+\mathrm{T}$ cell line that mediates murine interstitial nephritis. J. Clin. Invest. 88:408-416.

31. Neilson, E.G. 1993. The nephritogenic T lymphocyte response in interstitial nephritis. Semin. Nephrol. 13:496-502.

32. Kawasaki, K., Yaoita, E., Yamamoto, T., and Kihara, I. 1992. Depletion of CD8 positive cells in nephrotoxic serum nephritis of WKY rats. Kidney Int. 41:1517-1526.

33. Kurts, C., et al. 1996. Constitutive class I-restricted exogenous presentation of self antigens in vivo. J. Exp. Med. 184:923-930.

34. Bevan, M.J. 2004. Helping the CD8(+) T-cell response. Nat. Rev. Immunol. 4:595-602.

35. Behrens, G.M., et al. 2004. Helper requirements for generation of effector CTL to islet beta cell antigens. J. Immunol. 172:5420-5426.

36. Dudziak, D., et al. 2007. Differential antigen processing by dendritic cell subsets in vivo. Science. 315:107-111.

37. Burgdorf, S., Kautz, A., Bohnert, V., Knolle, P.A., and Kurts, C. 2007. Distinct pathways of antigen uptake and intracellular routing in CD4 and CD8 T cell activation. Science. 316:612-616.

38. Shortman, K., and Naik, S.H. 2007. Steady-state and inflammatory dendritic-cell development. Nat. Rev. Immunol. 7:19-30.

39. Villadangos, J.A., and Schnorrer, P. 2007. Intrinsic and cooperative antigen-presenting functions of dendritic-cell subsets in vivo. Nat. Rev. Immunol. 7:543-555.

40. Kurts, C., et al. 1997. CD4+ T cell help impairs $\mathrm{CD} 8+\mathrm{T}$ cell deletion induced by cross-presentation of self-antigens and favors autoimmunity. 
J. Exp. Med. 186:2057-2062.

41. Carbone, F.R., Kurts, C., Bennett, S.R., Miller, J.F., and Heath, W.R. 1998. Cross-presentation: a general mechanism for CTL immunity and tolerance. Immunol. Today. 19:368-373.

42. Harbers, S.O., et al. 2007. Antibody-enhanced crosspresentation of self antigen breaks $\mathrm{T}$ cell tolerance. J. Clin. Invest. 117:1361-1369.

43. Janssen, E.M., et al. 2003. CD4+ T cells are required for secondary expansion and memory in CD8+ T lymphocytes. Nature. 421:852-856.

44. Smith, C.M., et al. 2004. Cognate CD4(+) T cell licensing of dendritic cells in CD8(+) T cell immunity. Nat. Immunol. 5:1143-1148.

45. Redmond, W.L., and Sherman, L.A. 2005. Peripheral tolerance of CD8 T lymphocytes. Immunity. 22:275-284.

46. Calbo, S., et al. 2008. Functional tolerance of CD8+ T cells induced by muscle-specific antigen expression. J. Immunol. 181:408-417.

47. Na, S.Y., et al. 2008. Naive CD8 T-cells initiate spontaneous autoimmunity to a sequestered model antigen of the central nervous system. Brain. 131:2353-2365.

48. Wong, M.A., Cui, S., and Quaggin, S.E. 2000. Identification and characterization of a glomerular-specific promoter from the human nephrin gene. Am. J. Physiol. Renal Physiol. 279:F1027-F1032.

49. Ludwig-Portugall, I., Hamilton-Williams, E.E., Gottschalk, C., and Kurts, C. 2008. Cutting edge:
CD25+ regulatory $\mathrm{T}$ cells prevent expansion and induce apoptosis of B cells specific for tissue autoantigens. J. Immunol. 181:4447-4451.

50. Putaala, H., Soininen, R., Kilpelainen, P., Wartiovaara, J., and Tryggvason, K. 2001. The murine nephrin gene is specifically expressed in kidney, brain and pancreas: inactivation of the gene leads to massive proteinuria and neonatal death. Hum. Mol. Genet. 10:1-8.

51. Jung, S., et al. 2002. In vivo depletion of CD11c(+) dendritic cells abrogates priming of CD8 $(+) \mathrm{T}$ cells by exogenous cell-associated antigens. Immunity. 17:211-220.

52. Huang, H., et al. 2007. CD4+ Th1 cells promote CD8+ Tc1 cell survival, memory response, tumor localization and therapy by targeted delivery of interleukin 2 via acquired pMHC I complexes. Immunology. 120:148-159.

53. Randolph, G.J., Ochando, J., and Partida-Sanchez, S. 2008. Migration of dendritic cell subsets and their precursors. Annu. Rev. Immunol. 26:293-316.

54. Geissmann, F., et al. 2008. Blood monocytes: distinct subsets, how they relate to dendritic cells, and their possible roles in the regulation of T-cell responses. Immunol. Cell Biol. 86:398-408.

55. Castellino, F., et al. 2006. Chemokines enhance immunity by guiding naive CD8 $+\mathrm{T}$ cells to sites of CD4+ T cell-dendritic cell interaction. Nature. 440:890-895.

56. Lukacs-Kornek, V., et al. 2008. The kidney-renal lymph node-system contributes to cross-tolerance against innocuous circulating antigen. J. Immunol. 180:706-715.

57. Kurts, C., Kosaka, H., Carbone, F.R., Miller, J.F., and Heath, W.R. 1997. Class I-restricted crosspresentation of exogenous self-antigens leads to deletion of autoreactive CD8(+) T cells. J. Exp. Med. 186:239-245

58. Liu, K., Waskow, C., Liu, X., Yao, K., Hoh, J., and Nussenzweig, M. 2007. Origin of dendritic cells in peripheral lymphoid organs of mice. Nat. Immunol. 8:578-583.

59. Valenzuela, J., Schmidt, C., and Mescher, M. 2002. The roles of IL-12 in providing a third signal for clonal expansion of naive CD8 T cells. J. Immunol. 169:6842-6849.

60. Vielhauer, V., et al. 2003. Phenotyping renal leukocyte subsets by four-color flow cytometry: characterization of chemokine receptor expression. Nephron Exp. Nephrol. 93:e63.

61. Moeller, M.J., et al. 2004. Podocytes populate cellular crescents in a murine model of inflammatory glomerulonephritis. J. Am. Soc. Nephrol. 15:61-67.

62. Segerer, S., et al. 2008. Compartment specific expression of dendritic cell markers in human glomerulonephritis. Kidney Int. 74:37-46.

63. Panzer, U., et al. 2006. Compartment-specific expression and function of the chemokine IP-10/ CXCL10 in a model of renal endothelial microvascular injury. J. Am. Soc. Nephrol. 17:454-464. 